\title{
QUANTUM RESONANCES OF WEAKLY LINKED, MESOSCOPIC, SUPERCONDUCTING DOTS.
}

\author{
C.J. Lambert and A. Martin, \\ School of Physics and Materials \\ Lancaster University \\ Lancaster LA1 4YB \\ U.K.
}

\begin{abstract}
.
We examine quantum properties of mesoscopic, Josephson coupled superconducting dots, in the limit that charging effects and quantization of energy levels within the dots are negligible, but quasi-particle transmission into the weak link is not. We demonstrate that quasi-particle resonances lead to current-phase relations, which deviate markedly from those of weak links connecting macroscopic superconductors. Results for the steady state dc Josephson current of two coupled dots are presented.
\end{abstract}

PACS Numbers. 72.10.Bg, 73.40.Gk, 74.50. 
Recent theories of mesoscopic Josephson weak links[1,2] have revealed a variety of new quantization phenomena, which are absent from their macroscopic counterparts. For such systems, the size of the junction is less than or of order the phase breaking length, while the superconducting leads are macroscopic. In this Letter, we consider the quantum properties of weak links, formed when the superconductors themselves are mesoscopic. Linked mesoscopic, superconducting dots (LM dots), for which quasiparticles as well as Cooper pairs maintain phase coherence within the device, have been grown experimentally[3,4], although no theory of their quantum properties currently exists. In this Letter, we demonstrate that the current-phase relation of LM dots is sensitive to resonant tunneling of quasi-particles into the junction from external, current carrying leads. This leads to a non-equilibrium distribution of quasi-particles within the weak link, which is missing from conventional descriptions of Josephson junctions[5]. Such resonances, which lead us to view LM dots, as electronic analogues of Fabry-Perot interferometers, carry a current of order the critical current and therefore significantly modify the transport properties of such structures.

In a forthcoming publication[6] we shall present a complete theory capable of describing the steady state properties and slow dynamics of an arbitary number of LM dots. In this Letter we illustrate how scattering theory can be used to extract the key physics, by examining two such dots in one dimension, described by the Bogoliubov - de Gennes equation

$$
\mathbf{H}(\underline{r}) \underline{\Psi}(x)=E \underline{\Psi}(x),
$$

where

$$
\mathbf{H}=\left(\begin{array}{cc}
{\left[-\left(\hbar^{2} / 2 m\right) \partial_{x}^{2}+u(x)-\mu\right]} & \Delta(x) \\
\Delta^{*}(x) & -\left[-\left(\hbar^{2} / 2 m\right) \partial_{x}^{2}+u(x)-\mu\right]
\end{array}\right)
$$

In this equation, $\mu$ is the condensate chemical potential, $u(x)$ the normal scattering potential and $\Delta(x)$ the superconducting order parameter. A simple model of a pair a LM dots, shown in figure 1 , is obtained by allowing $\Delta(x)$ and $u(x)$ to be non-zero only 
in regions of size $L_{1}$ and $L_{2}$, where the order parameter phase takes values $\phi_{1}$ and $\phi_{2}$ respectively. Figure 1 shows a pair of LM dots connected by perfect, normal leads, to external reservoirs at chemical potentials $\mu_{a}$ and $\mu_{b}$ and distinguishes the present approach from other descriptions $[1,2,5]$, where the sources of charge are of no consequence and the lengths $L_{1}$ and $L_{2}$ are taken to be infinite. For LM dots, the system size $L_{1}+L+L_{2}$ is assumed to be smaller than the quasi-particle phase breaking length and therefore a description, which incorporates quasi-particle phase coherence throughout the device is appropriate.

To obtain such a description, consider the most general eigenstate of $\mathbf{H}$ belonging to eigen-energy $E$. In the regions where $\Delta(x)$ and $u(x)$ vanish, this has the form

$$
\underline{\Psi}(x)= \begin{cases}\left(\begin{array}{ll}
A \exp [i k x]+B \exp [-i k x] \\
C \exp [i q x]+D \exp [-i q x]
\end{array}\right), & \text { for } x<-\left(L_{1}+L / 2\right) ; \\
\left(\begin{array}{l}
A^{\prime \prime} \exp [i k x]+B^{\prime \prime} \exp [-i k x] \\
C^{\prime \prime} \exp [i q x]+D^{\prime \prime} \exp [-i q x]
\end{array}\right), & \text { for }-L / 2<x<L / 2 ; \\
\left(\begin{array}{l}
A^{\prime} \exp [i k x]+B^{\prime} \exp [-i k x] \\
C^{\prime} \exp [i q x]+D^{\prime} \exp [-i q x]
\end{array}\right), & \text { for }\left(L_{2}+L / 2\right)<x ;\end{cases}
$$

where $\hbar^{2} k^{2} / 2 m-\mu=\mu-\hbar^{2} q^{2} / 2 m=E$. In the absence of inelastic scattering, the quantum properties of such a structure can be described in terms of either a transfer matrix $T$ or scattering matrix $S$, defined by

$$
\left(\begin{array}{c}
O^{\prime} \\
I^{\prime}
\end{array}\right)=T\left(\begin{array}{c}
I \\
O
\end{array}\right) \quad \text { and } \quad\left(\begin{array}{c}
O \\
O^{\prime}
\end{array}\right)=S\left(\begin{array}{c}
I \\
I^{\prime}
\end{array}\right)
$$

where

$$
\left(\begin{array}{c}
O^{\prime} \\
I^{\prime}
\end{array}\right)=\hbar^{1 / 2}\left(\begin{array}{l}
k^{1 / 2} A^{\prime} \\
q^{1 / 2} D^{\prime} \\
k^{1 / 2} B^{\prime} \\
q^{1 / 2} C^{\prime}
\end{array}\right) \quad \text { (5a) } \quad \text { and } \quad\left(\begin{array}{c}
I \\
O
\end{array}\right)=\hbar^{1 / 2}\left(\begin{array}{c}
k^{1 / 2} A \\
q^{1 / 2} D \\
k^{1 / 2} B \\
q^{1 / 2} C
\end{array}\right)
$$

Once $T$ is known, $S$ can be constructed and vice versa[7,8]. Both $T$ and $S$ are functionals of all physical potentials, as well as functions of E. Since $\mathbf{H}$ is Hermitian, quasi-particle probability (though not charge) is conserved, and therefore $S$ is unitary. 
To describe the Josephson effect for such a structure, one must compute the current in the region $-L / 2<x<L / 2$, as well as in the external leads. To this end we form the expectation value of the current density operator, with respect to the density matrix corresponding to incident distributions $f_{i}^{\alpha}(E)$ of quasi-particles of type $\alpha$, energy $E$ along lead $i$, where $\alpha=+1(-1)$ for particles (holes). In the simplest case, $f_{i}^{\alpha}(E)$ would be a Fermi distribution, although the analysis outlined here can equally well describe transport properties arising from non-equilibrium (eg. hot electron) reservoir distributions. Expressions for the currents $I_{l}, I_{r}$ in the left and right leads respectively are written down in reference [9]. To obtain the current within the weak link, we introduce separate transfer matrices $T_{1}, T_{2}$ connecting plane wave amplitudes at $x=0$ to those in the left and right leads respectively and satisfying $T=T_{2} T_{1}$. The wavefunction $\underline{\Psi}(0)$ due to, for example, an incident particle from the left, is obtained by setting $A=1$, $D=B^{\prime}=C^{\prime}=0$ in equation (3) and using equation (4) to obtain the outgoing amplitudes $B$ and $C$. The corresponding coefficients $A^{\prime \prime}, B^{\prime \prime}, C^{\prime \prime}, D^{\prime \prime}$, which we denote $A_{1+}, \quad B_{1+}, \quad C_{1+}, \quad D_{1+}$, respectively are then obtained by acting on the vector (5b) with $T_{1}$. In this way, by combining the action of $S$ with $T_{1}$ and $T_{2}$, plane wave amplitudes $A_{i, \alpha}, \quad B_{i, \alpha}, \quad C_{i, \alpha}, \quad D_{i, \alpha}$ within the weak link, due to a quasi-particle of type $\alpha$, incident along lead $i$ can be constructed. For simplicity we restrict the present analysis to zero temperature, where the expectation value of the current density operator inside the weak link is found to be $I_{\mathrm{in}}=I_{s}+I_{q p}$, with

$$
I_{s}=(2 e / h) \sum_{i=1}^{2} \int_{0}^{\mu} d E\left\{\left[\left|D_{i-}\right|^{2}-\left|C_{i-}\right|^{2}\right]+[q(E) / k(E)]\left[\left|D_{i+}\right|^{2}-\left|C_{i+}\right|^{2}\right]\right\}
$$

and

$$
\begin{aligned}
I_{q p} & =(2 e / h) \int_{0}^{\mu_{a}-\mu} d E\left\{\left|A_{1+}\right|^{2}-\left|B_{1+}\right|^{2}+[q(E) / k(E)]\left[\left|C_{1+}\right|^{2}-\left|D_{1+}\right|^{2}\right]\right\} \\
& +(2 e / h) \int_{0}^{\mu-\mu_{b}} d E\left\{[k(E) / q(E)]\left[\left|A_{2-}\right|^{2}-\left|B_{2-}\right|^{2}\right]+\left|C_{2-}\right|^{2}-\left|D_{2-}\right|^{2}\right\}
\end{aligned}
$$

Note that the right hand side of equation (6) represents the contribution from all occupied negative energy states, which has been transformed, using the particle-hole symmetry 
relations $A_{i \alpha}(E)=D_{i-\alpha}^{*}(-E)$ and $B_{i \alpha}(E)=-C_{i-\alpha}^{*}(-E)$, to an integral over positive energies. The division of the total current into a sum of two currents is somewhat arbitrary. However if the reservoir potentials are equal, $I_{q p}$ vanishes, whereas $I_{s}$ may remain finite. Therefore in what follows, we refer to $I_{s}$ and $I_{q p}$ as the supercurrent and quasi-particle current respectively.

Before proceeding, it worth noting that in deriving equations (6) and (7), the occupancy of incoming states from external reservoirs has been chosen such that the ground state expectation value of any local operator is preserved by the transformation from a closed to an open system. More precisely, consider a scatter of size $L^{\prime}=L_{1}+L+L_{2}$ connected to leads of size $L^{\prime \prime}$, which join together to form a closed system of size $L^{\prime}+L^{\prime \prime}$, with periodic boundary conditions. If $\rho$ is the density matrix, then the expectation value $\operatorname{Tr} \rho O(x)$ of an operator $O(x)$ can be evaluated using any convenient set of basis states. For a closed system the obvious choice is the set of eigenstates of $\mathbf{H}$, satisfying periodic boundary conditions. However in the limit $L^{\prime \prime} \rightarrow \infty$, such a choice is no longer useful and a trace over all incoming scattering states is preferred. It is important to note that if the incoming scattering states are interpreted as arising from external reservoirs, then certain properties are imposed on the reservoirs by the scatterer. For example at zero temperature, in the absence of a potential difference, expectation values are preserved only if all incoming quasi-particle states are populated; both incoming electron and incoming hole states. Such occupancies are non-intuitive, since a more natural choice of zero temperature reservoirs would perhaps populate only incoming electron states. The relative merits of different choices of incoming distributions will be discussed in a forthcoming publication[6]. Here we merely note that the existence of quasi-particle resonances is independent of such choice, although the detailed form of the current-phase relation does depend on the distribution of incoming states.

As emphasized in reference [9], due to non-conservation of quasi-particle charge, the chemical potential $\mu$ enters expressions for $I_{l}$ and $I_{r}$ explicitly and in steady state, must 
be determined self-consistently by insisting that the currents are equal. In the present context one also notes that for an arbitrary phase difference $\phi=\phi_{1}-\phi_{2}$, the internal current $I_{\text {in }}$ will not equal the current in the leads. Hence to obtain a dc Josephson effect, in which a current flows between superconducting dots of equal potentials, both $\mu$ and $\phi$ must be determined from the steady state condition $I_{\text {in }}=I_{l}=I_{r}$. The solution to these equations yields a phase-current expression $\phi(I)$, which may be inverted to yield a more familiar current-phase relation $I(\phi)$. If the current supplied by the external reservoirs is greater than a certain value, it may happen that no solution to these equations exists, in which case a critical current $I_{c}$ has been exceeded. Since the primary aim of this Letter is to highlight the role of quasi-particle resonances, full implementation of this self-consistent scheme will be relegated to reference[6]. One notes however that for the symmetric structures considered below, the equation $I_{l}=I_{r}$ is trivially satisfied with the choice $\mu=\left(\mu_{a}+\mu_{b}\right) / 2$.

Except for a set of resonant energies, the quasi-particle contribution to $I_{\text {in }}$ is expected to be negligibly small, because for reservoir potentials less than typical values of $|\Delta(x)|$, quasi-particle states decay on the scale of the superconducting coherence length, which in practice may be much greater than $L_{1}$ and $L_{2}$. For a clean junction, in the limit $L_{1}, L_{2} \rightarrow \infty$, these resonances correspond to bound state energies of the weak link considered by Bardeen and Johnson[10]. In the latter description, the penetration of quasi-particles into the weak link is ignored and therefore in the clean limit, the analysis presented here reduces to that of reference[10] in the absence of quasi-particle transmission through the superconductors.

When solving equation (1), it is convenient to introduce a characteristic wavevector $k_{F}$ through the relation $\hbar^{2} k_{F}^{2} / 2 m=\mu$ and to divide both sides by $\mu$. The resulting equation depends only on the dimensionless quantities $\bar{E}=E / \mu, \bar{u}(x)=u(x) / \mu$, $\bar{\Delta}(x)=\Delta(x) / \mu$ and $\bar{x}=x k_{F}$. With this choice of scaling, equation (1) and therefore all scattering properties, do not depend explicitly on $\mu$ and it is convenient to express results 
in terms of the dimensionless potential differences $\bar{\mu}_{a}=\left(\mu_{a}-\mu\right) / \mu, \bar{\mu}_{b}=\left(\mu-\mu_{b}\right) / \mu$. To illustrate the effect of resonances on the current-phase relationship of LM dots, figure 2 shows plots of $I_{\text {in }}(\phi)$ versus $\phi$ for different choices of $\mu$. Choosing $|\bar{\Delta}(x)|=\bar{\Delta}_{0}$ in the superconducting regions of dimensionless length $\bar{L}_{1}, \bar{L}_{2}$, figure 2 shows results for the case $\bar{L}=100, \bar{L}_{1}=\bar{L}_{2}=150, \bar{\Delta}_{0}=0.04, \bar{u}(x)=0$ and an applied potential of $\bar{\mu}_{a b}=\left(\mu_{a}-\mu_{b}\right) / \mu=0.01$. These values are typical of those attainable experimentally, where $\bar{\Delta}_{0}$ is always much smaller than unity and the size of a dot is much greater than the coherence length $\bar{\xi}=\bar{\Delta}_{0}^{-1}$. The dotted, dashed and solid lines show results for $\bar{\mu}_{b}=0, \bar{\mu}_{a b} / 4$ and $\bar{\mu}_{a b} / 2$ respectively. The main part of the figure shows the internal current $I_{\text {in }}(\phi)=I_{s}(\phi)+I_{q p}(\phi)$, while the insert shows the quasi-particle current. Except near the endpoints, the supercurrent $I_{s}$ increases almost linearly with phase, as $\phi$ increases from $-\pi$ to $\pi$, reflecting the fact that under certain conditions, a phase difference across such a junction acts like a Galilean transformation[10]. In contrast $I_{q p}$ exhibits strong resonances over well defined intervals of $\phi$. In the limit $\bar{L}_{1}, \bar{L}_{2} \rightarrow \infty$, when $\bar{L}>>\bar{\Delta}_{0}^{-1}$, the bound state energies of such a junction occur at $[10] \bar{E}_{n} \simeq \pm \phi / \bar{L}+(2 n+1) \pi / \bar{L}$. As noted earlier these bound state energies become quasi-particle transmission resonances in a LM dots. For $\phi= \pm \pi$, such a resonance occurs at $E=0$ and therefore quasiparticles from external reservoirs can enter the weak link. To understand these results in more detail, consider first the quasi-particle current shown by the dashed line, arising when $\bar{\mu}_{b}=\bar{\mu}_{a b} / 4$ and therefore $\bar{\mu}_{a}=3 \bar{\mu}_{a b} / 4$. As $\phi$ increases from $\phi=-\pi$, the lowest resonant energy $\bar{E}_{0}$ first exceeds the highest incident quasi-particle energy $\bar{\mu}_{b}$ from the right reservoir, leading to a decrease in the quasi-particle current by a factor of $1 / 2$. As $\phi$ increase further, eventually the highest energy $\bar{\mu}_{a}$ of a quasi-particle from the left reservoir is exceeded and $I_{q p}$ switches off. For the case $\bar{\mu}_{a}=\bar{\mu}_{b}$ shown by the solid line, the quasi-particle currents from the two reservoirs switch off at the same phase, while for the case $\bar{\mu}_{b}=0$, only the left reservoir contributes to $I_{\mathrm{qp}}$.

It should be noted that the steps in $I_{\mathrm{qp}}$ are of order the critical current through 
the device, despite the fact that the superconductors are several coherence lengths long. This arises because resonant states within the weak link are formed from superpositions of particles and Andreev reflected holes, for which the currents add constructively. In a forthcoming publication, it will be shown that adding normal potential scatterers to the contacts between the external leads and superconductors does not significantly affect Andreev scattering within the weak link and therefore has only a marginal effect on the quasi-particle current, whereas potential scattering within the weak link suppresses both the supercurrent and the quasi-particle current.

Figure 2 illustrates the effect of quasi-particle resonances for one value of the external potential difference only. For larger values of $\mu_{a}-\mu_{b}$, more than one resonance can contribute and as shown by the solid lines of figure 3, the resulting internal current exhibits a non-trivial phase dependence. For completeness, the dashed lines show the external current in the leads and from the fact that these lines cross at several distinct values of $\phi$, one concludes that the fully self-consistent solution will possess many branches.

In this Letter, we have shown how current phase relations for LM dots can be obtained from a knowledge of the transfer matrices $T_{1}, T_{2}$ and the associated scattering matrix $S$. The results obtained highlight the role of quasi-particle resonances in determining junction properties. Figures 2 and 3 demonstrate that the quantum properties of LM dots can differ markedly from those of more conventional Josephson junctions, a feature which should manifest itself in a range of junction properties. A key property of the current-phase relations obtained in this Letter is that $I_{\mathrm{in}}(\phi)$ is no longer an odd function of $\phi$ and therefore its the phase average does not vanish. While one might be tempted to identify this phase average with a quasi-particle leakage current, it should be emphasized that it cannot be replaced by a phenomenological Ohmic term in a RSJ equation. Indeed in situations where the phase difference varies slowly with time, the quasi-particle current is transmitted as a series of pulses, reflecting the resonant nature of such electronic interferometers. 


\section{Acknowledgements.}

This work is supported by the SERC, the EC, NATO and the MOD. It has benefited from useful conversations with S.J. Robinson, B. Kramer, M Dykman, J-L. Pichard, V.C. Hui, J.H. Jefferson and F. Sols. Support from the Institute for Scientific Exchange is acknowledged. 


\section{Figure Captions.}

\section{Figure 1.}

The order parameter of a typical pair of LM dots, connected to external reservoirs at chemical potentials $\mu_{a}$ and $\mu_{b}$, with $\mu_{a}>\mu_{b}$.

\section{Figure 2.}

For $\bar{\mu}_{a}+\bar{\mu}_{b}=0.01$, this figure shows current-phase relations for a pair of LM dots of size $\bar{L}_{1}=\bar{L}_{2}=150$, separation $\bar{L}=100$ order parameter $\Delta_{0}=0.04$ and $u(x)=0$. The main body of the figure shows results for the total internal current $I_{\text {in }}(\phi)=I_{s}(\phi)+I_{q p}(\phi)$, arising when $\bar{\mu}_{b}=0$ (dotted line), $\bar{\mu}_{a b} / 4$ (dashed line) and $\bar{\mu}_{a b} / 2$ (solid line). The insert shows corresponding results for the quasi-particle current $I_{q p}(\phi)$ only.

Figure 3. For the same system used in figure 2, this figure shows the internal current $I_{\text {in }}(\phi)$ (solid curves) and the external current $I_{\text {ext }}(\phi)=I_{l}=I_{r}$ (dashed curves) for applied potential differences of magnitude $\bar{\mu}_{a b}=0.02$ (top left), 0.04 (top right), 0.06 (lower left) and 0.08 (lower right). 


\section{References.}

1. C.W. Beenakker, Phys. Rev. Lett. 673836 (1991).

2. L.I. Glazman and K.A. Matveev, Sov. Phys.-JETP Lett. 49659 (1989).

3. V.T. Petrashov and V.N. Antonov, Sov. Phys. JETP Lett. 54 241, (1991)

4. V.T. Petrashov, V.N. Antonov, P. Delsing and T. Claeson, Phys. Rev. Lett. 70347 (1993).

5. K.K. Likharev, Rev. Mod. Phys. 51101 (1979).

6. C.J. Lambert, A. Martin and S.J. Robinson, to be published.

7. C.J. Lambert, V.C. Hui and S.J. Robinson, J.Phys.: Condens. Matter, 54187 (1993).

8. B. Kramer, Festkörperprobleme: Adv. Sol. St. Phys. 3053 (Vieweg, Braunschweig 1990).

9. C.J. Lambert, J. Phys.: Condens. Matter, 36579 (1990).

10. J. Bardeen and J.L. Johnson, Phys. Rev. B5 72 (1972). 